SUBJECT AREAS:

MULTIPLE SCLEROSIS

FUNCTIONAL MAGNETIC RESONANCE IMAGING

FATIGUE

MOTOR CONTROL

Received

22 April 2013

Accepted

28 May 2013

Published

18 June 2013

Correspondence and requests for materials should be addressed to

M.B. (marco.bove@ unige.it)

* These authors contributed equally to this work.

\section{The fatigue-motor performance paradox in multiple sclerosis}

\author{
Matteo Pardini ${ }^{1,2 *}$, Laura Bonzano ${ }^{1,2 *}$, Luca Roccatagliata ${ }^{2,3}$, Giovanni L. Mancardi ${ }^{1,2}$ \& Marco Bove ${ }^{4}$
}

'Department of Neuroscience, Rehabilitation, Ophthalmology, Genetics, Maternal and Child Health, University of Genoa, Genoa, Italy, ${ }^{2}$ Magnetic Resonance Research Centre on Nervous System Diseases, University of Genoa, Genoa, Italy, ${ }^{3}$ Department of Health Sciences, University of Genoa, Genoa, Italy, ${ }^{4}$ Department of Experimental Medicine, Section of Human Physiology and Centro Polifunzionale di Scienze Motorie, University of Genoa, Genoa, Italy.

Subjective fatigue is a typical symptom in Multiple Sclerosis (MS) even in the earliest stages of the disease. The relationship between persistent fatigue and motor task performance is still unclear. Aim of this study was to better investigate this relationship at both the motor behavioral and neuroanatomical levels. Towards this goal, we combined a quantitative evaluation of an undemanding finger motor task with concurrent brain functional magnetic resonance imaging (fMRI) in a group of MS patients with minimal disability but reporting persistent subjective fatigue. We found an unexpected significant positive correlation between persistent subjective fatigue and task-related temporal accuracy, revealing a "fatigue-motor performance paradox". fMRI analysis indicated that this association is potentially mediated by cerebellar and orbitofrontal cortex activity, suggesting a role of these regions in developing subjective fatigue. Our data point to a possible adaptive role for fatigue as the subjective correlate of increased resource demand for motor activities.

ubjective fatigue is a typical symptom of neurological diseases, such as Multiple Sclerosis (MS), Parkinson's disease and stroke ${ }^{1}$. Fatigue is reported by $60-90 \%$ of patients with $\mathrm{MS}^{2}$ and is characterized by the sensation of loss of physical and mental energy. Fatigue may develop at any stage of MS and is unrelated to disability status $^{3,4}$. There is at present limited understanding of the neural basis of fatigue perception. In recent years, converging evidence pointed to the prefrontal and deep grey matter structures as two key players in fatigue in patients with MS, as shown by the modulation of their activity by fatigue levels and by the relationship between increased fatigue and structural or functional alterations in these regions ${ }^{4-7}$.

Despite these advances, different key issues remain unsolved in current models of fatigue. To date, the relationship between persistent subjective fatigue and motor task performance is still unclear. Performance reduction due to a neurological condition could represent: i) a predisposing factor in the development of persistent fatigue, ii) a consequence of persistent fatigue, or iii) an unrelated phenomenon.

Here, we combined a quantitative evaluation of an undemanding finger motor task with concurrent brain functional magnetic resonance imaging (fMRI) in a group of MS patients presenting with persistent subjective fatigue. Aim of this study was to evaluate the relationship between persistent fatigue perception and task performance at both the motor behavioral and neuroanatomical levels.

\section{Results}

Patients were asked to perform a finger motor sequence repetition with the right (dominant) hand, metronomepaced at $2 \mathrm{~Hz}$ during a 2-min fMRI session, according to a boxcar design with two 30-s task periods alternating with two 30-s rest periods (see Methods).

All patients completed the experimental session without reporting task-induced fatigue.

Temporal accuracy (TA, i.e., the ability to keep up the metronome rhythm) values were significantly lower than $80 \%$, that is the lowest value usually observed in healthy subjects ${ }^{8}$. However, we found a significant positive relationship between total Modified Fatigue Impact Scale (MFIS) score and TA ( $r=0.92 ; \mathrm{p}<0.0001)$ (Fig. 1a), showing that increased levels of persistent subjective fatigue were associated with higher TA. It is worthwhile to highlight that subjective fatigue evaluation was performed before the task and was focused on the perception of fatigue in the previous month and not related to task performance. This unpredicted finding could, at a first glance, be akin of a "fatigue-motor performance paradox".

From the fMRI analysis, we observed that the motor task performance mainly activated the left Brodmann's Area (BA)2, BA3, BA4 and BA6 and the right cerebellum (Fig. 1b), as commonly observed when healthy subjects 


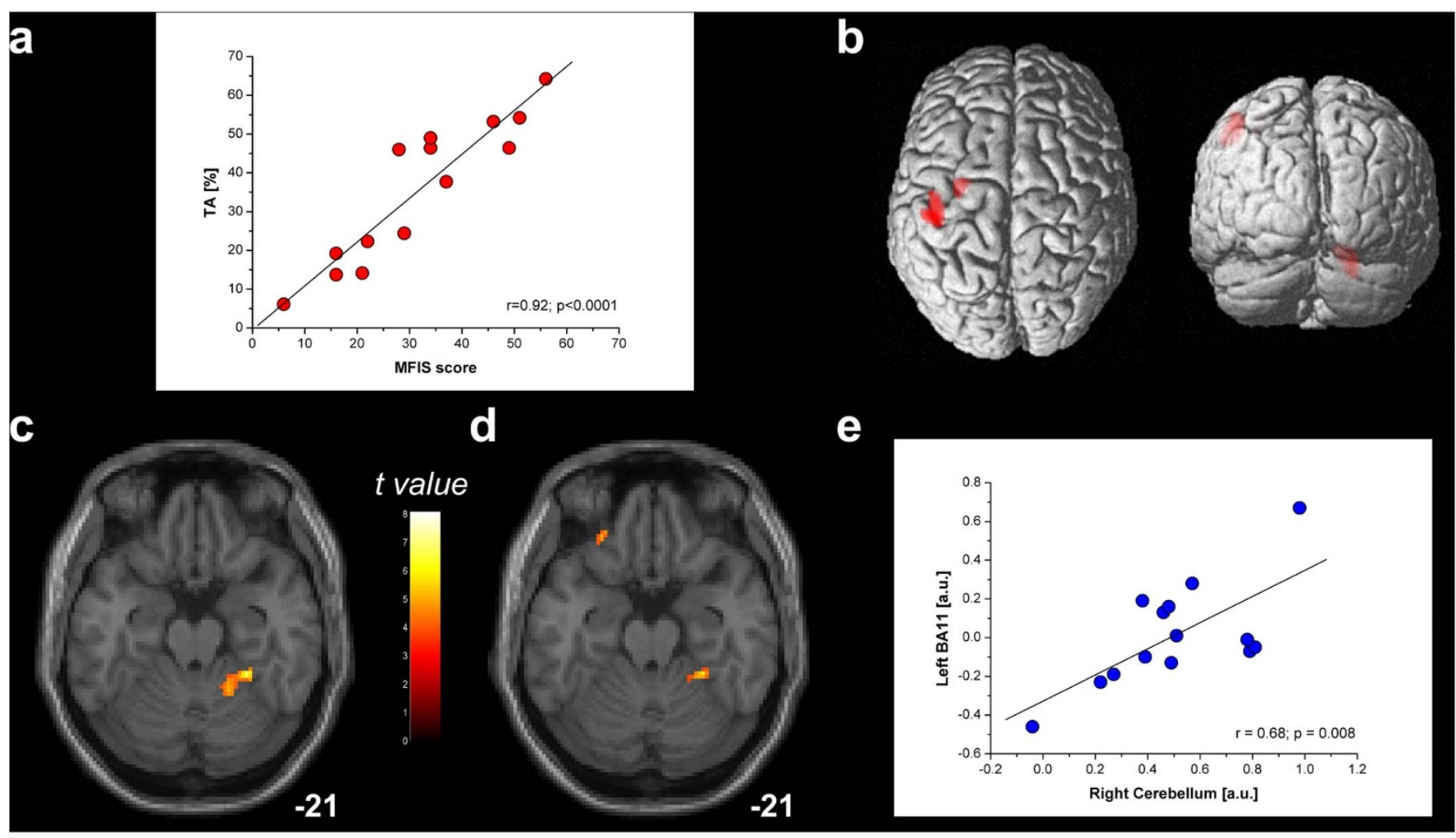

Figure $1 \mid$ Behavioral and fMRI analysis during a finger motor sequence repetition with the right hand at $2 \mathrm{~Hz}$. (a) Positive correlation between Modified Fatigue Impact Scale (MFIS) score and temporal accuracy (TA) ( $\mathrm{r}=0.92 ; \mathrm{p}<0.0001)$, indicating that patients with higher day-to-day fatigue score showed increased TA compared with less fatigued patients. (b) Task-related group activation map ( $\mathrm{p}<0.05$ FWE corrected; size $=50$ voxels) for the finger-to-thumb opposition sequence. (c) Regression analysis with TA as covariate. A positive correlation was observed in the right cerebellar hemisphere (cluster $\mathrm{p}=0.001$; size $=225$ voxels), indicating that patients with higher TA showed increased cerebellar activity. (d) Regression analysis with MFIS score as covariate. A positive relationship with the right cerebellar hemisphere (cluster $\mathrm{p}=0.041$; size $=65$ voxels) and the left orbitofrontal cortex (OFC; cluster $\mathrm{p}=0.045$; size $=62$ voxels) indicated that patients with higher perceived persistent fatigue showed more active prefrontal and cerebellar areas. (e) Linear relationship between the 1st-eigenvariate extracted in the TA-related activation in the right cerebellum and in the MFIS-related activation in the left OFC $(\mathrm{r}=0.68, \mathrm{p}=0.008)$ [arbitrary units].

perform a finger motor task $\mathrm{k}^{9}$. Further, the regression model conducted on the fMRI data with TA as covariate revealed that only the right cerebellar hemisphere positively correlated with increasing TA (MNI peak coordinate $=20 ;-52 ;-18$; cluster $\mathrm{p}=0.001$; maximum $-\mathrm{t}=8.08$; cluster extent $=225$ voxels; Fig. $1 \mathrm{c}$ ). This finding is in line with the known role played by the cerebellum in the performance of acoustically-paced finger motor sequences ${ }^{10}$ and with the hypothesis that the cerebellum is engaged during tasks requiring the precise representation of temporal information ${ }^{11}$.

Taking into account MFIS score as covariate, a positive relationship with the right cerebellar hemisphere and the left BA11orbitofrontal cortex (OFC) was found (right cerebellum: MNI peak coordinate $=26 ;-40 ;-22$; cluster $\mathrm{p}=0.041$; maximum $-\mathrm{t}=8.44$; cluster extent $=65$ voxels; OFC: MNI peak coordinate $=-26 ; 36$; -18 ; cluster $\mathrm{p}=0.045$; maximum-t $=5.42$; cluster extent $=62$ voxels; Fig. 1d) indicating that patients with higher perceived persistent fatigue showed more active prefrontal and cerebellar areas. Moreover, the MFIS-related OFC activation positively correlated with the TA-related cerebellar activation $(\mathrm{r}=0.68, \mathrm{p}=0.008)$ (Fig. 1e), suggesting a functional interaction between these two areas.

\section{Discussion}

Our fMRI results hint to a possible explanation for the observed positive relationship between persistent fatigue and task-related temporal accuracy. Converging evidence points to a key role for the OFC in fatigue perception. Task-induced fatigue, for example, was shown to modulate OFC activity in healthy subjects ${ }^{12,13}$. Moreover, the OFC is connected with prefrontal and deep structures known to mediate sensorimotor processing, motivation and self-evaluation ${ }^{14,15}$, which are known to play a significant role in fatigue ${ }^{1}$. Cerebellar activations, on the other hand, were shown to be related not only with TA but also with MFIS score and to represent the only common area between the TA-related and the MFIS-related activation patterns (Figs. 1c, d). The observed relationship between persistent fatigue and taskrelated TA could represent an epiphenomenon of the shared activation pattern between MFIS and TA or the functional connectivity of OFC and cerebellar areas ${ }^{16}$.

In this context, our data add to previous findings showing a correlation between motor performance during a brief and simple motor task and persistent subjective fatigue, suggesting a potential adaptive role for fatigue. While our current experimental construct does not allow to disentangle the cause-effect relationship between increased fatigue perception and increased temporal accuracy, fatigue seems to represent the experiential correlate of the increased resource demand for motor activities ${ }^{17}$, potentially acting as an adaptive fail-safe mechanism to avoid exhaustive states.

Lastly, from the methodological point of view, our data point to the usefulness of the integration of objective motor performance evaluation, cognitive testing and fMRI data to study complex constructs such as fatigue. Summing up, our findings show an unexpected highly-significant positive correlation between increased persistent fatigue and task-related temporal accuracy in MS, potentially mediated by OFC and cerebellar activity. These data expand the current models of fatigue perception suggesting a possible explanation of the adaptive role of subjective fatigue. 
Statistics. Pearson's correlation coefficients were calculated to evaluate the

\section{Methods}

Patients. Fourteen patients with relapsing-remitting Multiple Sclerosis (MS) and minimal disability (Expanded Disability Status Scale - EDSS $^{18} \leq 2$ ) were included in this study $(7$ females and 7 males, mean age $=45.9 \pm 7.8$ years, mean disease duration $=11.8 \pm 5.9$ years, mean EDSS $=1.5 \pm 0.5)$. These patients were recruited consecutively, among the patients with MS presenting to our MS Centre subjectively reporting fatigue as important symptom affecting their quality of life. Patients' history was negative for relapses, changes in the EDSS score or corticosteroids use in the six months preceding the study. Patients with medical or psychiatric causes of fatigue including sleep disorders were not considered eligible for the study. All the enrolled patients presented with routine laboratory tests within normal ranges including thyroid-stimulating hormone essay and were free from psychoactive drug use. Moreover all patients underwent a neuropsychological evaluation to exclude the presence of conditions that could affect the self-report of perceived fatigue (i.e., significant cognitive deficits, mood disorders or alexithymia).

Screening for cognitive deficits was performed with the Symbol Digit Modalities Test (SDMT), a measure of attention and processing speed ${ }^{19}$. The SDMT is thought to present good specificity and sensitivity for MS-related cognitive deficits even in the early stages of the disease ${ }^{20}$.

Moreover, all the enrolled patients presented with SDMT scores higher that the $5^{\text {th }}$ percentile of the SDMT normative data, that is the accepted SDMT cut-off value (SDMT z score $=-0.12 \pm 0.75)$ to exclude the presence of clinically-relevant cognitive impairment ${ }^{21}$. They were evaluated for the presence of co-morbid depression using the Structured Clinical Interview for DSM-IV-TR Axis I Disorders ${ }^{22}$ and the Hospital Anxiety and Depression Scale (HADS). The HADS is a self-report rating scale used to evaluate depression and anxiety in subjects with medical conditions ${ }^{23}$. All the included patients scored in the normal range (i.e., lower than 8) in both HADS subscales (HADS Depression $=1.93 \pm 0.61$; HADS Anxiety $=1.14 \pm 0.77$ )

The reduced capacity to describe emotions and inner states was assessed with the Toronto Alexytimia Scale - 20 (TAS-20). The obtained scores were within the normal range for the TAS 20 (TAS- $20=30.2 \pm 1.9)^{24}$.

Fatigue was assessed with the Modified Fatigue Impact Scale (MFIS), a modified form of the Fatigue Impact Scale ${ }^{25}$, that is a self-report questionnaire in which higher scores represent higher levels of perceived fatigue (range 0-64). Although all the patients came to medical attention for significant self-reported chronic fatigue (i.e., a qualitative perception of an inner state), the MFIS allowed to quantify the impact of fatigue on daily activities and revealed a large range of the distribution of MFIS scores in the group (mean MFIS $=31.8 \pm 14.9$ ).

All the enrolled patients were right-handed according to a modified Italiantranslated Edinburgh Handedness Inventory ${ }^{26}$ and naive to the specific purpose of this study. Informed consent was obtained according to the policy of our Institutional Review Board and to the Declaration of Helsinki.

MRI acquisition. Each patient underwent brain MRI scanning with structural and fMRI sequences covering the whole brain, performed on a 1.5 Tesla MR system (Signa Excite, General Electric Healthcare, WI, USA). The MRI protocol included axial T2weighted imaging (slice thickness $=5 \mathrm{~mm} ; \mathrm{TR}=6300 \mathrm{~ms} ; \mathrm{TE}=123.7 \mathrm{~ms} ; \mathrm{FOV}=$ $260 \times 260 \mathrm{~mm}$; matrix $=256 \times 256$ ), used as structural reference for the fMRI acquisition. Functional MRI data were acquired with axial T2*-weighted single-shot spin-echo echoplanar imaging (slice thickness $=5 \mathrm{~mm} ; \mathrm{TR}=3000 \mathrm{~ms} ; \mathrm{TE}=40 \mathrm{~ms}$; $\mathrm{FOV}=260 \times 260 \mathrm{~mm}$; matrix $=64 \times 64)$.

fMRI experiment. For each patient we acquired one fMRI run based on a block paradigm alternating two 30-s task periods with two 30-s rest periods.

During the rest periods, the patients were instructed to stay at rest without making any overt movement. The motor task consisted in the repetition of a finger-to-thumb opposition sequence with the right (dominant) hand (index, medium, ring and little fingers). The finger motor sequence was paced by a metronome set at $2 \mathrm{~Hz}$. We focused on a brief task requiring low-medium attention load $(2 \mathrm{~Hz}$ is about the spontaneous rate in healthy subjects ${ }^{8}$ ), to reduce confounding effects of high taskrelated effort on brain activity. We were able to measure finger motor performance concomitantly with fMRI acquisition by an ad hoc developed MR-compatible engineered glove ${ }^{12}$. The acoustic cue was delivered to the patients inside the MRI room through pneumatic headphones (Stereo Builder's Kit, Scan Sound, Inc., FL, USA) during the whole fMRI acquisition, also in the rest periods, to maintain the same acoustic stimulation. Patients were asked to keep their eyes closed during the whole experimental session, to avoid possible confounding effects due to the integration of acoustic and visual information during the task and to avoid visual cortical activation. Each fMRI session lasted 2:09 min; the first three brain volumes (i.e., 9 s) were discarded to allow steady-state magnetization.

Behavioral data analysis. The raw data recorded by the glove system were processed with an ad hoc software tool. We assessed temporal accuracy (TA) by calculating the percentage of touches preceding the metronome tone over the total number of correct touches. Movement anticipation with respect to an acoustic signal has been indicated as a physiological mechanism necessary to perceive the movement in synchrony with the cue at a central representation level ${ }^{27}$. The percentage of advance movements was found to be greater than $80 \%$ in healthy subjects performing the same finger motor sequence at $2 \mathrm{~Hz}^{8}$.

Particularly, we calculated the average of the motor performance parameter on the two 30 -s blocks, thus obtaining a single mean value of TA for each patient on the total of touches performed in 1-min period. relationships of MFIS with the motor performance parameter (TA).

SPM5 software (Wellcome Department of Imaging Neuroscience, London, UK) was used for fMRI processing and statistical analysis, as described elsewhere ${ }^{28}$. For each patient, after movement correction and slice timing, the functional images were realigned to the first functional image, normalized to the Montreal Neurological Institute (MNI) template brain image using a 12-parameter affine transformation, re sampled to $2 \times 2 \times 2 \mathrm{~mm}^{3}$ voxels and smoothed with an $8 \mathrm{~mm}$ full-width at halfmaximum isotropic Gaussian kernel to increase the signal-to-noise ratio. A genera linear model was used to identify the voxels with task-related signal changes at the individual level. Task-related t contrast images were created for each patient and then introduced into a second-level random-effect analysis to allow for population inferences. The corresponding group activation map was determined using one-sample ttest with a height threshold of $\mathrm{p}<0.05$ FWE corrected and a minimum cluster size arbitrarily set to 50 voxels.

We then implemented two second-level analyses with a regression model based on the single task-related $t$ contrast images, independently considering the TA values and MFIS scores as covariates. For each contrast we obtained a group activation map related to the behavioral covariate (i.e., temporal accuracy and fatigue perception, respectively) with a height threshold of $\mathrm{p}<0.001$ uncorrected and minimum cluster size of 50 voxels.

The first eigenvariates of the BOLD signal in the clusters surviving each secondlevel analysis were extracted with the eigenvariate procedure implemented in SPM and the relationship between the values obtained in the cluster located in the orbitofrontal cortex (defined in the MFIS-related analysis) and those in the cerebellar cluster (defined in the TA-related analysis) was evaluated with a parametric correlation approach.

Data in the text are reported as mean \pm SD.

1. Chaudhuri, A. \& Behan, P. O. Fatigue in neurological disorders. Lancet 363, 978-988 (2004)

2. Krupp, L. B., Alvarez, L. A., LaRocca, N. G. \& Scheinberg, L. C. Fatigue in multiple sclerosis. Arch Neurol 45, 435-437 (1988).

3. Cook, D. B., O'Connor, P. J., Lange, G. \& Steffener, J. Functional neuroimaging correlates of mental fatigue induced by cognition among chronic fatigue syndrome patients and controls. Neuroimage 36, 108-122 (2007).

4. DeLuca, J., Genova, H. M., Hillary, F. G. \& Wylie, G. Neural correlates of cognitive fatigue in multiple sclerosis using functional MRI. J Neurol Sci 270, 28-39 (2008).

5. Inglese, M. et al. Deep gray matter perfusion in multiple sclerosis: dynamic susceptibility contrast perfusion magnetic resonance imaging at 3 T. Arch Neurol 64, 196-202 (2007).

6. Pardini, M., Bonzano, L., Mancardi, G. L. \& Roccatagliata, L. Frontal networks play a role in fatigue perception in multiple sclerosis. Behav Neurosci 124, 329-336 (2010).

7. Roelcke, U. et al. Reduced glucose metabolism in the frontal cortex and basal ganglia of multiple sclerosis patients with fatigue: a $18 \mathrm{~F}$-fluorodeoxyglucose positron emission tomography study. Neurology 48, 1566-1571 (1997).

8. Bove, M. et al. The effects of rate and sequence complexity on repetitive finger movements. Brain Res 1153, 84-91 (2007).

9. Stoodley, C. J. \& Schmahmann, J. D. Functional topography in the human cerebellum: a meta-analysis of neuroimaging studies. Neuroimage 44, 489-501 (2009).

10. Rao, S. M. et al. Distributed neural systems underlying the timing of movements. J Neurosci 17, 5528-5535 (1997).

11. Ivry, R. B. \& Spencer, R. M. The neural representation of time. Curr Opin Neurobiol 14, 225-232 (2004).

12. Bonzano, L. et al. Basal ganglia are active during motor performance recovery after a demanding motor task. Neuroimage 65, 257-266 (2013).

13. Tajima, S. et al. Medial orbitofrontal cortex is associated with fatigue sensation. Neurol Res Int 2010, 671421 (2010).

14. Boksem, M. A. \& Tops, M. Mental fatigue: costs and benefits. Brain Res Rev 59, 125-139 (2008).

15. Cavada, C., Company, T., Tejedor, J., Cruz-Rizzolo, R. J. \& Reinoso-Suarez, F. The anatomical connections of the macaque monkey orbitofrontal cortex. A review. Cereb Cortex 10, 220-242 (2000).

16. Krienen, F. M. \& Buckner, R. L. Segregated fronto-cerebellar circuits revealed by intrinsic functional connectivity. Cereb Cortex 19, 2485-2497 (2009).

17. Wallis, J. D. Neuronal mechanisms in prefrontal cortex underlying adaptive choice behavior. Ann N Y Acad Sci 1121, 447-460 (2007).

18. Kurtzke, J. F. Rating neurologic impairment in multiple sclerosis: an expanded disability status scale (EDSS). Neurology 33, 1444-1452 (1983).

19. Smith, A. Symbol Digit Modalities Test (SDMT): Manual (Western Psychological Services, Los Angeles, CA, 1982).

20. Deloire, M. S. et al. How to detect cognitive dysfunction at early stages of multiple sclerosis? Mult Scler 12, 445-452 (2006).

21. Amato, M. P. et al. The Rao's Brief Repeatable Battery and Stroop Test: normative values with age, education and gender corrections in an Italian population. Mult Scler 12, 787-793 (2006).

22. First, M. B., Spitzer, R. L., Gibbon, M. \& Williams, J. Research Version, Patient Edition. (SCID-I/P) (New York State Psychiatric Institute, New York, 2002). 
23. Zigmond, A. S. \& Snaith, R. P. The hospital anxiety and depression scale. Acta Psychiatr Scand 67, 361-370 (1983).

24. Bagby, R. M., Parker, J. D. \& Taylor, G. J. The twenty-item Toronto Alexithymia Scale--I. Item selection and cross-validation of the factor structure. J Psychosom Res 38, 23-32 (1994).

25. Fisk, J. D. et al. Measuring the functional impact of fatigue: initial validation of the fatigue impact scale. Clin Infect Dis 18 Suppl 1, S79-83 (1994).

26. Oldfield, R. C. The assessment and analysis of handedness: the Edinburgh inventory. Neuropsychologia 9, 97-113 (1971).

27. Aschersleben, G. Temporal control of movements in sensorimotor synchronization. Brain Cogn 48, 66-79 (2002).

28. Friston, K. J. et al. Analysis of fMRI time-series revisited. Neuroimage 2, 45-53 (1995)

\section{Acknowledgements}

We thank M. Vettraino for excellent technical assistance. This work was supported by the Italian Ministry of Health (RF 2007 n. 6/2008).

\section{Author contributions}

M.P., L.B. and M.B. conceived and designed the experiments. M.P., L.B. and L.R. performed the experiments. M.P., L.B. and M.B. analyzed the data. M.P., L.B. and M.B. interpreted the data and wrote the paper. L.R. and G.L.M. drafted the article or revised it critically for important intellectual content.

\section{Additional information}

Competing financial interests: The authors declare no competing financial interests.

How to cite this article: Pardini, M., Bonzano, L., Roccatagliata, L., Mancardi, G.L. \& Bove, M. The fatigue-motor performance paradox in multiple sclerosis. Sci. Rep. 3, 2001; DOI:10.1038/srep02001 (2013).

(c) (i) (5) $\Theta$ This work is licensed under a Creative Commons Attribution-

NonCommercial-NoDerivs 3.0 Unported license. To view a copy of this license, visit http://creativecommons.org/licenses/by-nc-nd/3.0 